\title{
Corrigendum to "Comparison between Fluconazole with Oral Protexin Combination and Fluconazole in the Treatment of Vulvovaginal Candidiasis"
}

\author{
S. Nouraei, ${ }^{1}$ S. Amir Ali Akbari, ${ }^{2}$ M. Jorjani, ${ }^{3}$ H. Alavi Majd, ${ }^{4}$ M. Afrakhteh, ${ }^{5}$ \\ A. Ghafoorian, ${ }^{6}$ and H. Tafazzoli Harandi ${ }^{1}$ \\ ${ }^{1}$ Faculty of Nursing and Midwifery, Shahid Beheshti University of Medical Sciences, International Branch, Tehran, Iran \\ ${ }^{2}$ Department of Midwifery, Faculty of Nursing and Midwifery, Shahid Beheshti University of Medical Sciences, Tehran, Iran \\ ${ }^{3}$ Department of Pharmacology and Neuroscience, Research Center, Shahid Beheshti University of Medical Sciences, Tehran, Iran \\ ${ }^{4}$ Department of Biostatistics, Shahid Beheshti University of Medical Sciences, Tehran, Iran \\ ${ }^{5}$ Department of Obstetrics \& Gynecology, Shahid Beheshti University of Medical Sciences, Tehran, Iran \\ ${ }^{6}$ Shahid Beheshti University of Medical Sciences, Tehran, Iran \\ Correspondence should be addressed to S. Amir Ali Akbari; sedaliakbari@gmail.com
}

Received 17 September 2017; Accepted 9 October 2017; Published 27 November 2017

Copyright (C) 2017 S. Nouraei et al. This is an open access article distributed under the Creative Commons Attribution License, which permits unrestricted use, distribution, and reproduction in any medium, provided the original work is properly cited.

In the article titled "Comparison between Fluconazole with Oral Protexin Combination and Fluconazole in the Treatment of Vulvovaginal Candidiasis," [1] there was an error in the Abstract section, where " $\left(\chi^{2}=0.01, P=6.7\right)$ " should be corrected to " $\left(\chi^{2}=6.7, P=0.01\right)$ ".

\section{References}

[1] S. Nouraei, S. Amir Ali Akbari, M. Jorjani et al., "Comparison between fluconazole with oral protexin combination and fluconazole in the treatment of vulvovaginal candidiasis," ISRN Obstetrics and Gynecology, vol. 2012, Article ID 375806, 10 pages, 2012. 


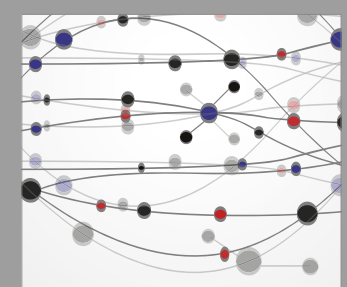

The Scientific World Journal
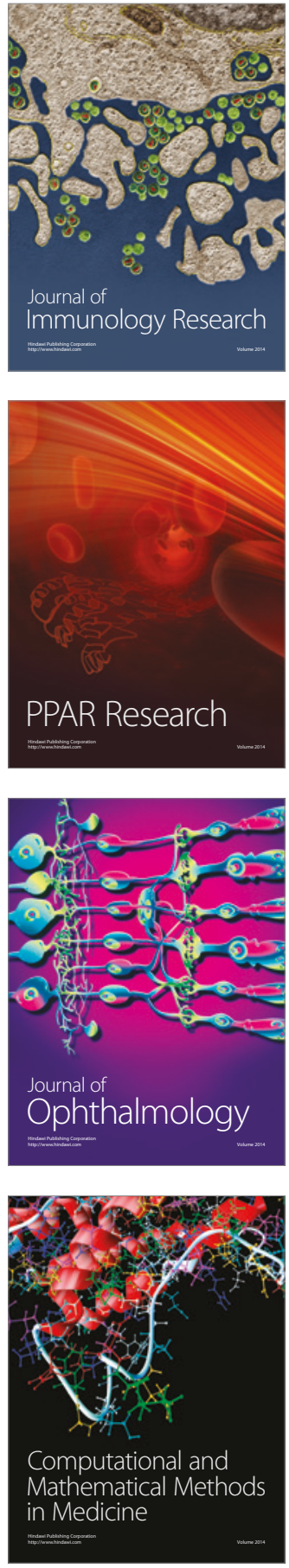

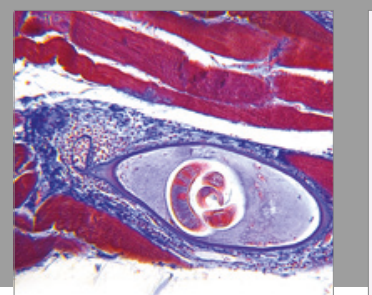

Gastroenterology Research and Practice
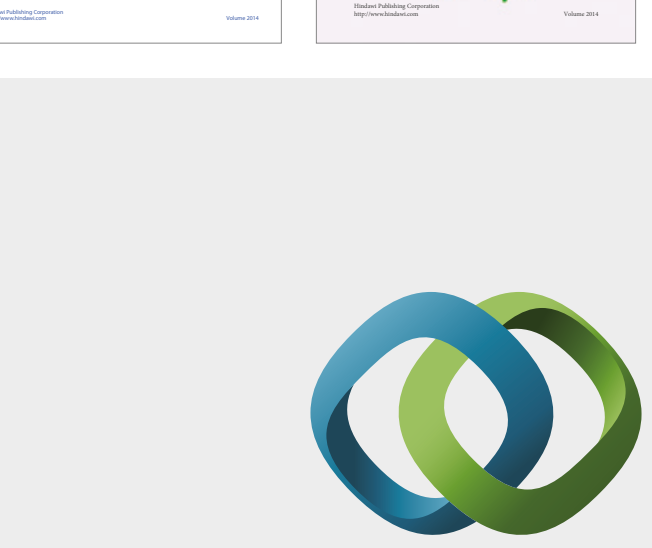

\section{Hindawi}

Submit your manuscripts at

https://www.hindawi.com
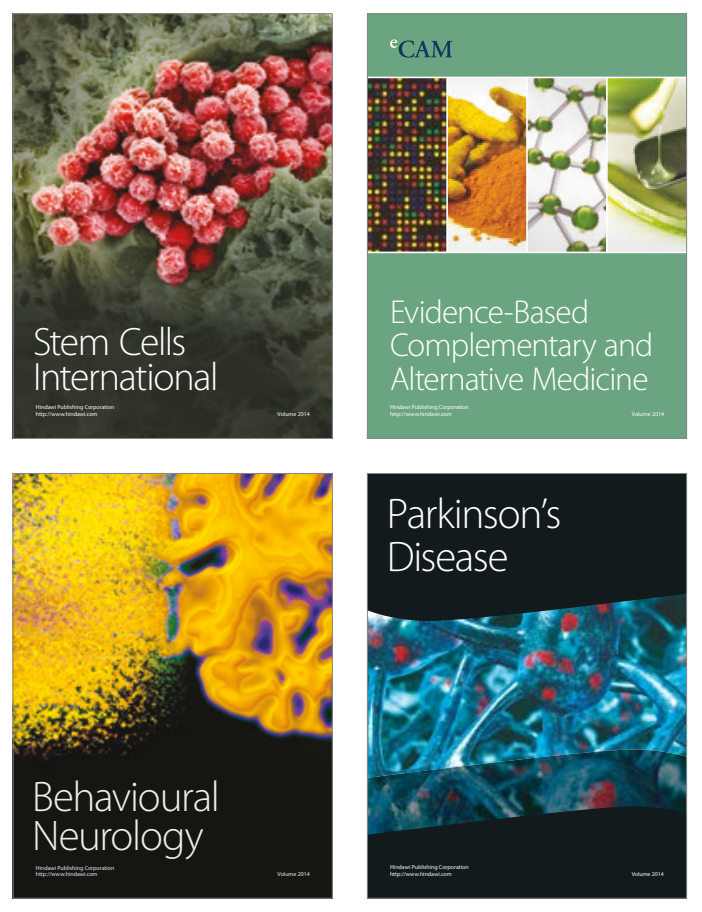
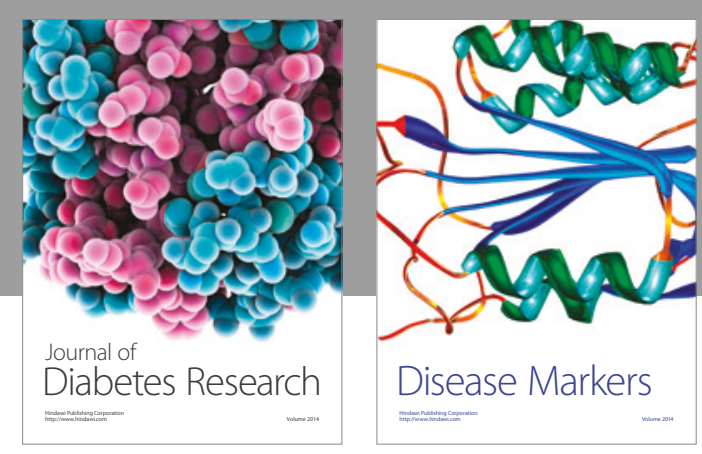

Disease Markers
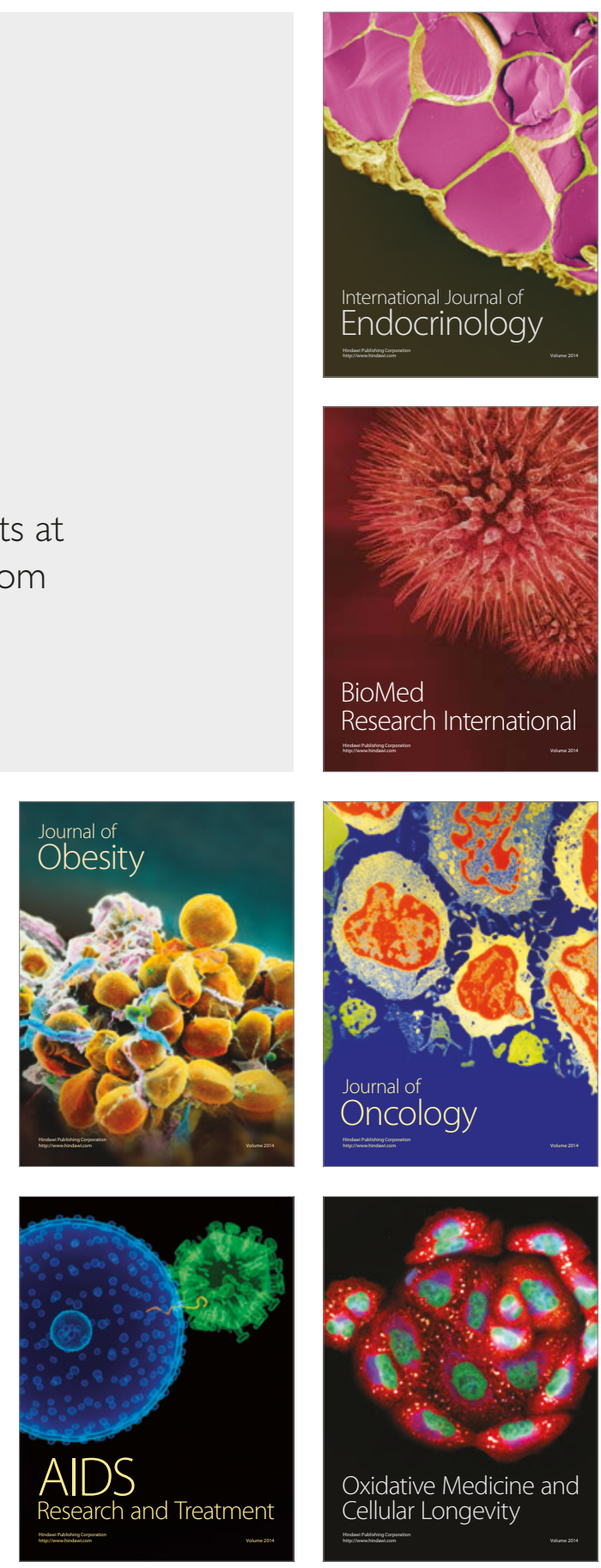